\title{
Monolithic and hybrid integration of InAs/GaAs quantum dot microdisk lasers on silicon
}

Kryzhanovskaya, N., Moiseev, E., Nadtochiy, A., Maximov, M., Dragunova, A., et al.

N. Kryzhanovskaya, E. Moiseev, A. Nadtochiy, M. Maximov, A. Dragunova, M. Fetisova, M. Kulagina, Yu. Guseva, S. Mintairov, N. Kalyuzhnyy, M. Tang, M. Liao, J. Wu, S. Chen, H. Liu, A. Zhukov, "Monolithic and hybrid integration of InAs/GaAs quantum dot microdisk lasers on silicon," Proc. SPIE 11775, Integrated Optics: Design, Devices, Systems and Applications VI, 117750P (18 April 2021); doi: 10.1117/12.2589118

SPIE. Event: SPIE Optics + Optoelectronics, 2021, Online Only 


\title{
Monolithic and hybrid integration of InAs/GaAs quantum dot microdisk lasers on silicon
}

\author{
N. Kryzhanovskaya ${ }^{* a, b}$, E. Moiseev ${ }^{\mathrm{a}}$, A. Nadtochiy ${ }^{\mathrm{a}}$, M. Maximov $^{\mathrm{b}, \mathrm{a}}$, A. Dragunova ${ }^{\mathrm{a}}$, M. Fetisova ${ }^{\mathrm{b}}$, \\ M. Kulagina ${ }^{\mathrm{c}}$, Yu. Guseva ${ }^{\mathrm{c}}$, S. Mintairov ${ }^{\mathrm{c}}$, N. Kalyuzhnyy ${ }^{\mathrm{c}}$, M. Tang ${ }^{\mathrm{d}}$, M. Liao ${ }^{\mathrm{d}}, \mathrm{J}_{\text {. Wu }}^{\mathrm{d}}$, S. Chen $^{\mathrm{d}}$, \\ H. Liu ${ }^{\mathrm{d}}$, A. Zhukov ${ }^{\mathrm{a}, \mathrm{b}}$

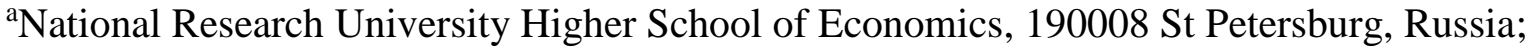 \\ ${ }^{b}$ Alferov University, 194021 St Petersburg, Russia; \\ 'Ioffe Institute, St Petersburg, 194021, Russia; \\ dUniversity College London, London, WC1E 7JE, UK
}

\begin{abstract}
A method of hybrid integration of quantum dot microdisk lasers with silicon wafer is proposed and realized. In addition to the possibility of combining microlasers with various silicon-based electronic and photonic devices, this makes it possible to significantly improve heat removal from the active region of the microlaser. The thermal resistance normalized to the mesa area reaches the level of about $0.002(\mathrm{~K} / \mathrm{W})^{*} \mathrm{~cm}^{2}$, which is significantly lower than the corresponding values of QD microlasers on GaAs substrate and monolithically grown on Si. As a result, the threshold current as well as current-induced shift of emission wavelength are reduced in continuous-wave regime.
\end{abstract}

Keywords: Semiconductor laser, microdisk laser, nanostructures, hybrid integration

\section{INTRODUCTION}

Strong localization of charge carriers within individual quantum dots (QDs) makes them less sensitive to non-radiative recombination caused by either epitaxy-related defects or by semiconductor surface since the carrier diffusion is suppressed [1]. Owing to this unique properties, self-organized QDs did confirm their potential for using as an active region of light-emitting devices fabricated by means of deep etching, e.g. microdisk/microring lasers, and/or made of epitaxial materials with high dislocation densities, e.g. III-V heterostructures directly grown on silicon [2-4]. QD-based microlasers grown on GaAs offer better temperature stability compared to their InP-based counterparts [5,6]. For example, GaAs-based QD microdisk lasers have revealed their ability to low-threshold lasing even at 100 ${ }^{\circ} \mathrm{C}$ [7].

Hybrid integration of III-V microlasers with silicon is also attracting significant interest as an alternative to monolithic integration. Separate fabrication of a III-V microlaser or an array of the microlasers and their subsequent transfer to a foreign substrate greatly simplify the compatibility of the epitaxial growth of a laser material with the process of manufacturing Si-based electronic and photonic components. The residual GaAs substrate can be used as a common (-) electrode to the array of microlasers, whereas separate current injection into the selected laser element is provided by the formation of electrically isolated (+) contact pads on the surface of Si or SOI substrate, Fig. 1, a. Light outcoupling from the microlaser can be carried out into free space or into ridge waveguides made on the surface. Moreover, placing microlasers $p$-side down should improve their thermal resistance as compared to conventional ( $p$-side up) geometry. It is of great importance since the self-heating effect causes an additional increase of the threshold current and sets the minimum diameter of the microlaser at which the laser can operate in $\mathrm{CW}$ regime [8].

In the present work we describe a method suitable for integration of QD microdisk laser with silicon and present the results of the preliminary characterization of hybrid-integrated QD microlasers.

*nataliakryzh@gmail.com; phone+7-812-6445911; https://spb.hse.ru/

Integrated Optics: Design, Devices, Systems and Applications VI, edited by Pavel Cheben, Jiř́ Čtyroký,

Iñigo Molina-Fernández, Proc. of SPIE Vol. 11775, 117750P · C 2021 SPIE

CCC code: $0277-786 \mathrm{X} / 21 / \$ 21 \cdot$ doi: $10.1117 / 12.2589118$

Proc. of SPIE Vol. $11775117750 \mathrm{P}-1$ 


\section{EXPERIMENTAL METHODS}

A laser heterostructure was grown by low-pressure metal-organic chemical vapor deposition on an n+-doped GaAs substrate slightly misoriented off (100) plane. The substrate misorientation together with a moderate InAs mole fraction $(\mathrm{x} \sim 40 \%)$ and some other specific growth regimes promotes formation of thickness and compositional modulation of InGaAs layer. Thicker regions having higher indium content can be considered as an ensemble of island referred to as quantum well-dots (QWDs) [9]. The surface density of the QWD islands is about (3..5) $\times 10^{11} \mathrm{~cm}^{-2}$, whereas for the conventional Stranski-Krastanow quantum dots a density of $5 \times 10^{10} \mathrm{~cm}^{-2}$ is typical. We found that higher density of QDs results in more efficient extraction of light of whispering gallery modes into free space due probably to enhanced mode scattering at the microcavity sidewalls. This peculiarity of QWD-based microdisk resonators significantly simplifies their characterization by optical methods.

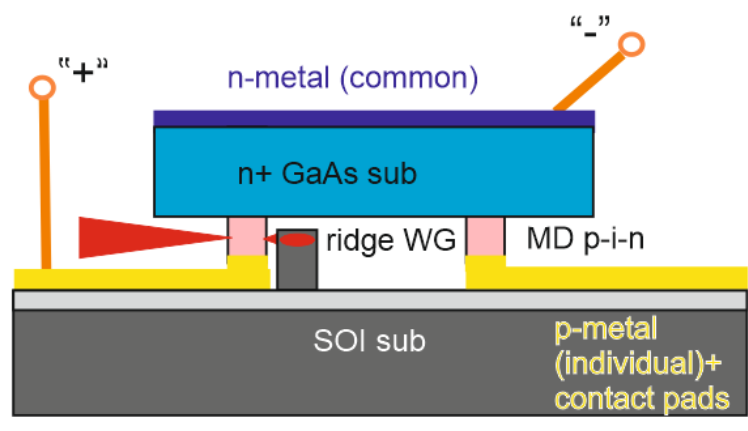

a

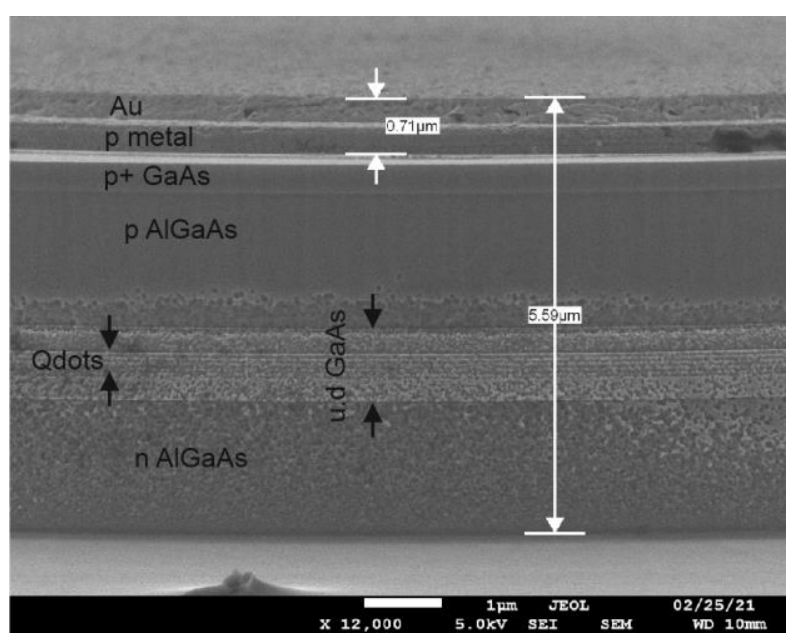

b

Figure 1. Sketch of microdisk array transferred to foreign substrate comprising functional elements on its surface (a) and scanning electron micrograph of laser heterostructure taken at microdisk sidewall (b).

The laser active region comprises several QWD planes placed in the middle of a GaAs waveguiding layer jointly sandwiched between n-type and p-type $\mathrm{Al}_{0.3} \mathrm{Ga}_{0.7} \mathrm{As}$ cladding layers, Fig. 1,b. Microdisk resonators were formed by dry etching of circular mesas with a height of about $6 \mu \mathrm{m}$ and different diameters varied from 10 to $50 \mu \mathrm{m}$. The etching was performed through the active region so that the mesa height was about $7 \mu \mathrm{m}$. No sidewall passivation was used. Upon p+ GaAs cap layer of the laser heterostructure, round-shaped $\mathrm{AgMn} / \mathrm{Ni} / \mathrm{Au}$ contacts were formed individually to each microdisk. After that the GaAs substrate was thinned down to $\sim 100 \mu \mathrm{m}$, and a join n-type contact was put onto a back side of the substrate. After that, the wafer was diced into chips containing various (up to 12) microdisks.

We used high-resistive (>1000 Ohm/cm) silicon substrate to transfer microlaser chips onto it. $\mathrm{Cr} / \mathrm{Au}$ contact pads were formed on the silicon surface using laser lithography and lift-off process. The geometry of the contact pads and conductive tracks between them provided the possibility of individual or group electrical connection to microlasers, Fig. 2 ,a. The pad dimensions were chosen to correspond to the diameters of the top contacts of the microlasers. The tolerance of $0.5 \mu \mathrm{m}$ was realized.

Fig. 2,b shows the microlasers attached to a silicon wafer. One can clearly see a decrease in the thickness of the intermediate layers between the microlasers and the surface on which they are located due to the mounting method we used. The thickness decreases from $\sim 100$ to less than $10 \mu \mathrm{m}$. Microlaser positioning and bonding was performed with FINEPLACER ${ }^{\circledR}$ lambda 2 (Finetech, Germany). In contrast to our previous work [10], in which the indium-gold eutectic was used for fixing the microlaser chip on the Si wafer, in the present work we used gold preforms, Fig. 3,a. The used bonding regimes (temperature and force) ensure reliable attachment of microdisks to the wafer and, at the same time, their electrical connection. The method ensures that the III-V chip are sufficiently firmly attached to the silicon substrate. In particular, we found that when the chip is torn off, the semiconductor material is split in the microdisk area, while the place where the microcircuit is attached to the substrate does not break, Fig. 3,b. 


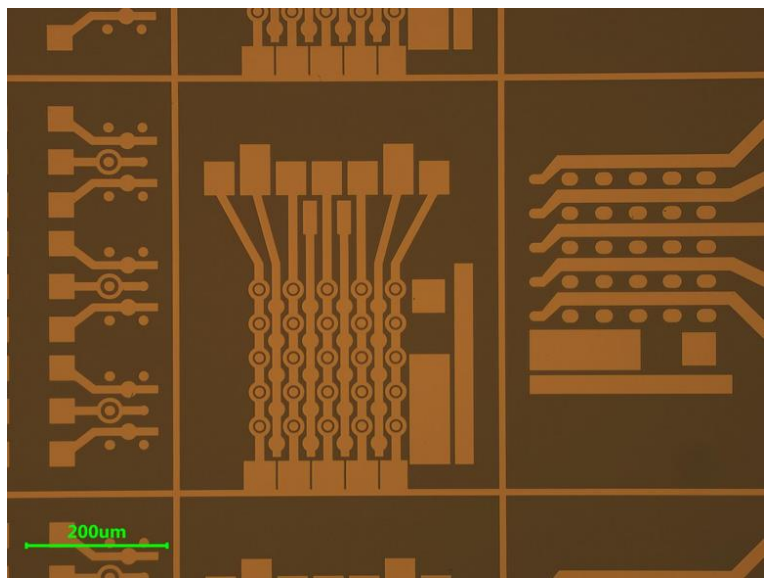

a

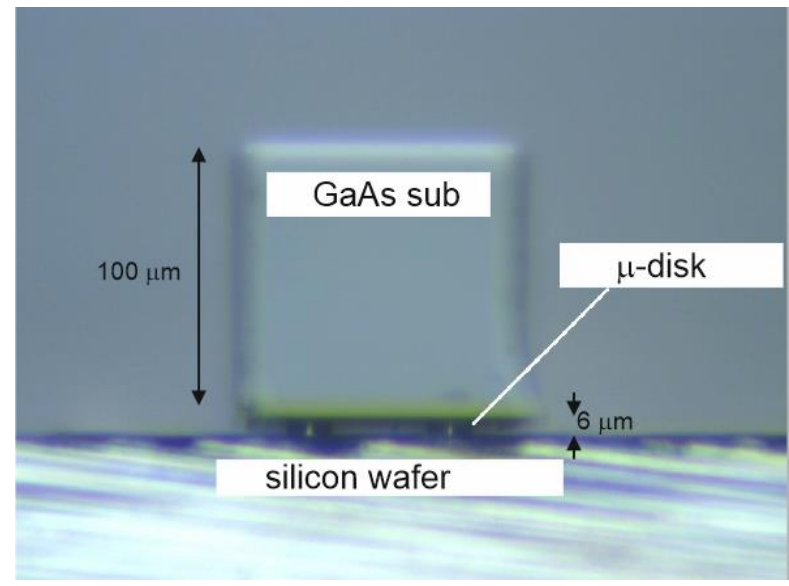

b

Figure 2. Microscopic image of contact pads and conductive tracks on silicon wafer (a) and side view of microdisk chip attached to silicon substrate (b).

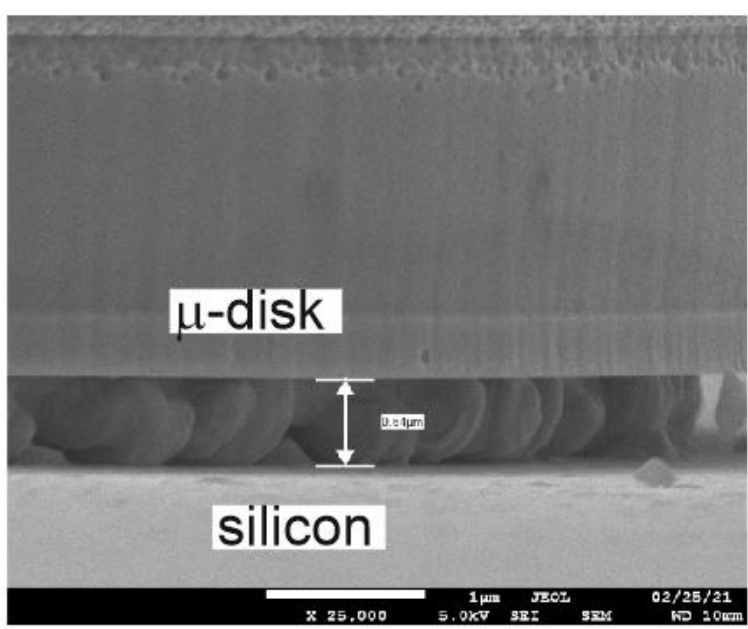

a

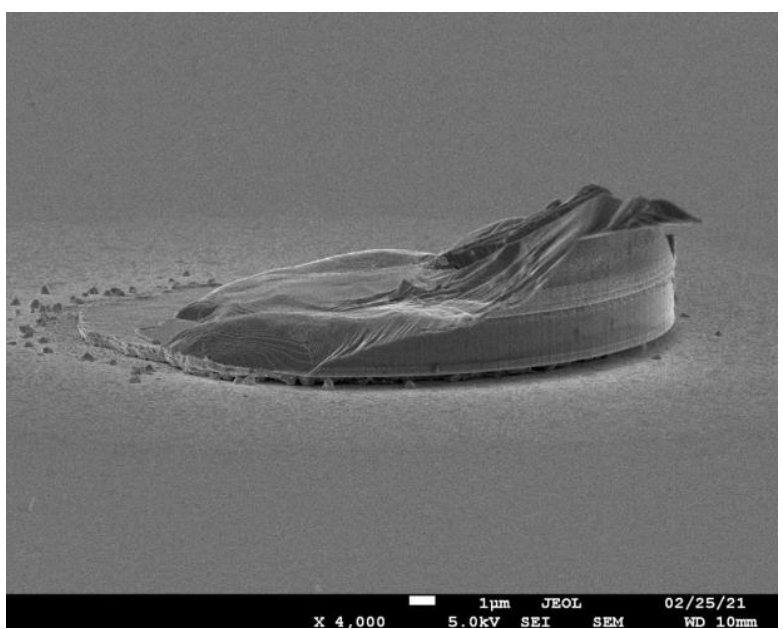

$\mathrm{b}$

Figure 3. Close-up image of the gap between silicon surface and microdisk with gold preforms (a) and scanning electron microscope image of the microdisk after its detachment from silicon (b).

\section{RESULTS AND DISCUSSION}

Microlasers were tested at room temperature in continuous wave regime. Neither cooler nor heatsinking was used. We did not observe any change of emission spectra of QDs caused by the structure annealing during bonding. Fig. 4,a, where current-voltage characteristics are presented, reveals that bonding the microdisk chip on the non-native substrate does not affect the electrical performance. Meanwhile we found that the threshold current is noticeably decreases after the microlaser transfer onto silicon. In Fig. 4.b, where the coupled to the fiber light power of microlaser is shown as function of injection current, the threshold current of $25 \mathrm{~mA}$ can be estimated for the initial microlaser and $17 \mathrm{~mA}$ for the microlaser bonded to silicon.

We believe that the improvement of the threshold current in hybridly integrated microlasers is associated with better heat removal from the active region. The microlaser thermal resistance was evaluated from the experimental data of the wavelength red-shift against the power consumed, these data are presented in Fig. 5,a. The slope decreases in the example presented from 0.033 to $0.027 \mathrm{~nm} / \mathrm{K}$, which corresponds to the improvement of the microlaser thermal resistance by more than 20\%. Fig. 5,b summarizes thermal resistance data for QD microdisk lasers of different types. 
The discussed hybrid integrated microlasers provide the thermal resistance-area product of about $2.2 \times 10^{-3}(\mathrm{~K} / \mathrm{W}) \mathrm{x} \mathrm{cm}^{2}$, which is more than 2 times lower than the corresponding values for microlasers monolithically integrated with $\mathrm{Si}$.

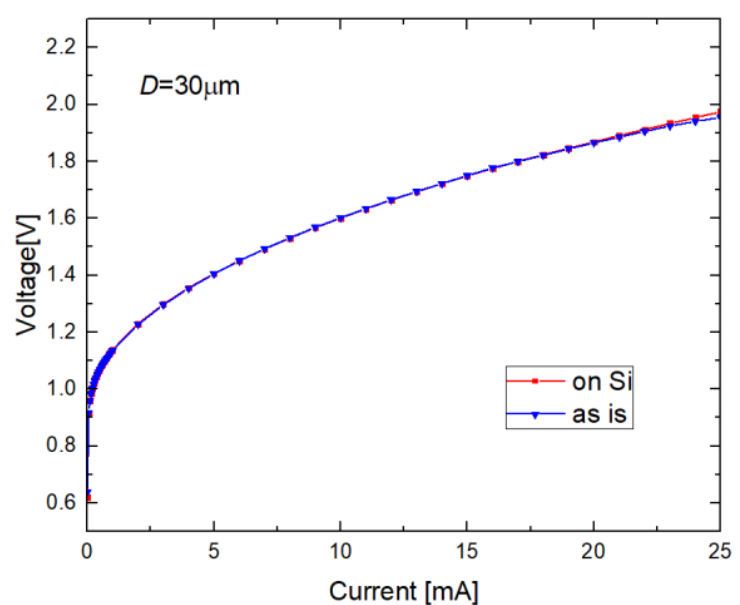

a

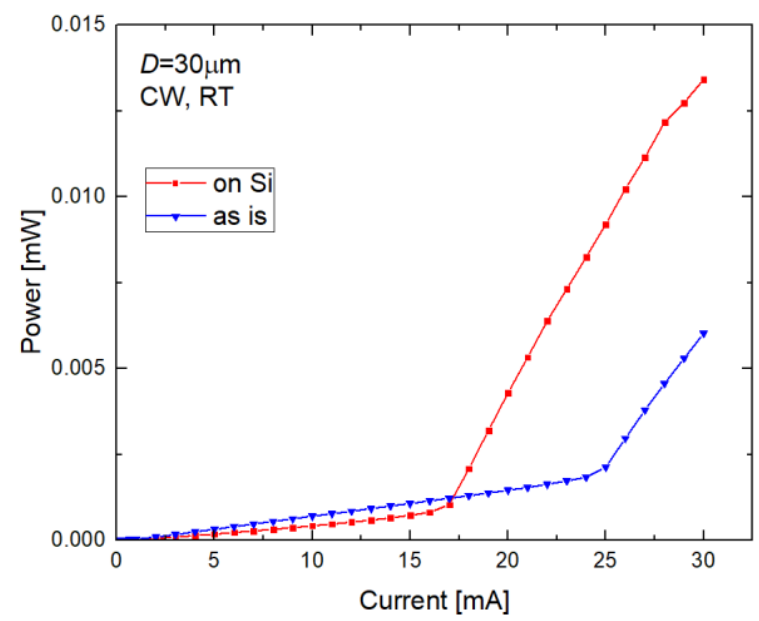

$\mathrm{b}$

Figure 4. Examples of current-voltage (a) and light-current (b) characteristics of microdisk lasers: as-is and after integration with silicon wafer.

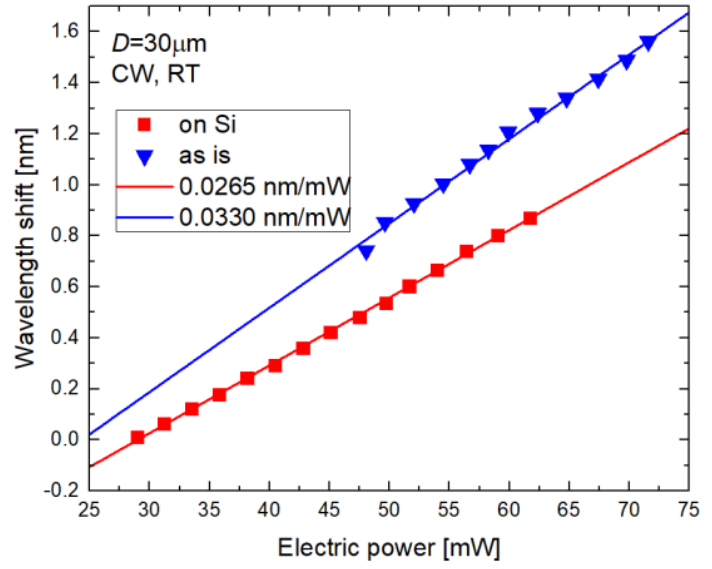

a

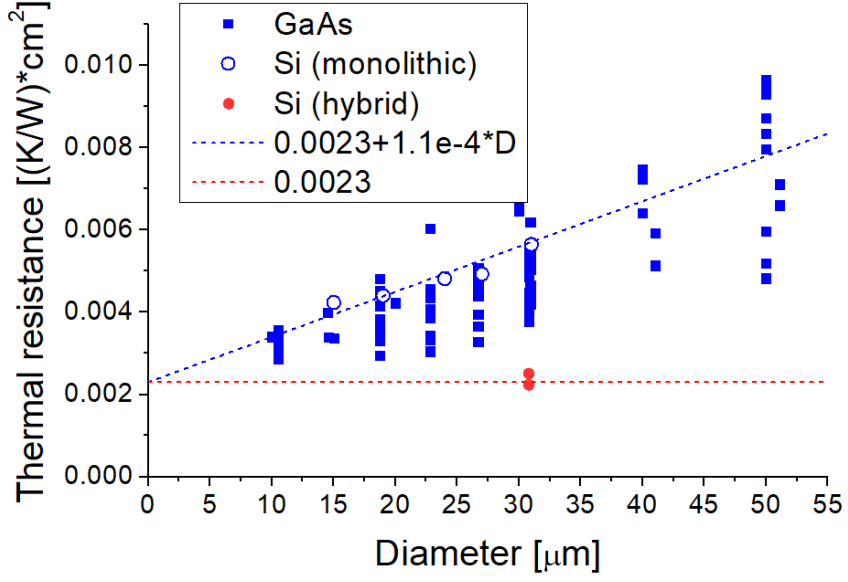

b

Figure 5. Red shift of lasing wavelength as function of consumed electric power of as-is mirodisk laser and after its integration with silicon wafer (a); thermal resistance of microdisk lasers of various type against the diameter (b).

\section{CONCLUSION}

In conclusion, a method of hybrid integration of InAs/InGaAs/GaAs quantum dot micodisks lasers to silicon is demonstrated. Both single microlasers and microlaser arrays can be transferred to silicon with opportunity of individual electric connection. Owing to a reduction of the thickness between the microlaser active region and the surface in p-side down bonded microlasers, the thermal resistance is greatly improved as compared to initial devices, where the GaAs substrate additionally impedes the heat dissipation. Lower thermal resistance leads to less significant self-heating of the microlaser. In it turn, this results in lowering the threshold current and higher stability of lasing wavelength. Lowering the energy-to-data ration under high speed modulation of hybrid integrated microlasers is expected. 


\section{ACKNOWLEDGEMENTS}

The work was supported by the Russian Science Foundation (19-72-30010). Support of optical measurements from the Basic Research Program of the National Research University Higher School of Economics is gratefully acknowledged.

\section{REFERENCES}

[1] Ouyang, D., Ledentsov, N. N., Bimberg, D., Kovsh, A. R., Zhukov, A. E., Mikhrin, S. S. and Ustinov, V. M., "High performance narrow stripe quantum-dot lasers with etched waveguide", Semicond. Sci. Technol. 18(12) L53-L54 (2003).

[2] Wan, Y., Norman, J., Li, Q., Kennedy, M. J., Liang, D., Zhang, C., Huang, D., Zhang, Z., Liu, A. Y., Torres, A., Jung, D., Gossard, A. C., Hu, E. L., Lau, K. M. and Bowers, J. E., "1.3 $\mu$ m submilliamp threshold quantum dot micro-lasers on Si”, Optica 4(8), 940-944 (2017).

[3] Kryzhanovskaya, N., Moiseev, E., Polubavkina, Yu., Maximov, M., Kulagina, M., Troshkov, S., Zadiranov, Yu., Guseva, Yu., Lipovskii, A., Tang, M., Liao, M., Wu, J., Chen, S., Liu, H. and Zhukov, A., "Heatsink-free $\mathrm{CW}$ operation of injection microdisk lasers grown on Si substrate with emission wavelength beyond $1.3 \mu \mathrm{m}$ ", Opt. Lett. 42(17), 3319-3322 (2017).

[4] Wan, Y., Inoue, D., Jung, D., Norman, J. C., Shang, C., Gossard, A.C. and Bowers, J.E., "Directly modulated quantum dot lasers on silicon with a milliampere threshold and high temperature stability", Photon. Res. 6(8), 776-781 (2018).

[5] Zou, L.-X., Huang, Y.-Z., Liu, B.-W., Lv, X.-M., Ma, X.-W., Yang, Y.-D., Xiao, J.-L. and Du, Y., "Thermal and high speed modulation characteristics for AlGaInAs/InP microdisk lasers" Opt. Express 23(3), 2879 (2015).

[6] Zhang, C., Liang, D., Kurczveil, G., Bowers, J. E. and Beausoleil, R. G., "Thermal management of hybrid silicon ring lasers for high temperature operation”, IEEE J. Selected Topics Quantum Electron. 21(6), 15026071502607 (2015).

[7] Moiseev, E., Kryzhanovskaya, N., Maximov, M., Zubov, F., Nadtochiy, A., Kulagina, M., Zadiranov, Yu., Kalyuzhnyy, N., Mintairov, S., and Zhukov, A. "Highly efficient injection microdisk lasers based on quantum well-dots", Opt. Lett. 43(19), 4554-4557 (2018).

[8] Zhukov, A. E., Kryzhanovskaya, N. V., Moiseev, E. I., Nadtochiy, A. M., Dragunova, A. S., Maximov, M. V., Zubov, F. I., Kadinskaya, S. A., Berdnikov, Yu., Kulagina, M. M., Mintairov, S. A., and Kalyuzhnyy, N. A. "Impact of self-heating and elevated temperature on performance of quantum dot microdisk lasers", IEEE J. Quantum Electron. 56(5), 2000908 (2020).

[9] Mintairov, S. A., Kalyuzhnyy, N. A., Maximov, M. V., Nadtochiy, A. M., Rouvimov, S. and Zhukov, A. E. "GaAs quantum well-dots solar cells with spectral response extended to $1100 \mathrm{~nm}$ ", Electron. Lett. 51(20), 1602-1604 (2015).

[10]Zhukov, A. E., Kryzhanovskaya, N. V., Moiseev, E. I., Dragunova, A. S., Tang, M., Chen, S., Liu, H., Kulagina, M. M., Kadinskaya, S. A., Zubov, F. I., Mozharov, A. M., and Maximov, M. V. "InAs/GaAs quantum dot microlasers formed on silicon using monolithic and hybrid integration methods", Materials 13(10), 2315-1-14 (2020). 